\title{
Current role of breast duct microendoscopy in malignancy
}

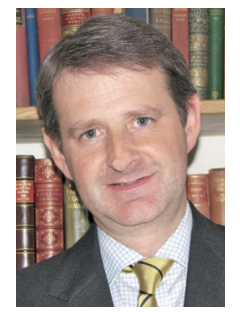

"There is now convincing evidence that breast duct microendoscopy provides an effective and noninvasive way to make a more definitive diagnosis in patients with nipple discharge."

\section{Nicolas Beechey-Newman}

The Harley Street Breast Clinic, 148 Harley Street, London, W1G 7LG, UK = Tel.: +44 2079086071 - Fax: +44 2079356423 m nbn@sky.com

Nipple discharge is the third most common symptom that provokes referral to a breast clinic after lump and breast pain. It accounts for approximately $5-7 \%$ of all women undergoing investigation for primary breast symptoms, but using current diagnostic methods is still a problem that is usually difficult to diagnose with certainty. The most common causes of nipple discharge are periductal mastitis, a chronic sterile inflammatory condition of the proximal ducts and duct papilloma, but in some series up to $11 \%$ of cases are due to malignancy $[1,2]$. Periductal mastitis typically presents as bilateral viscous, multiduct, multicolored (sometimes an almost pathognomonic dark green) nipple discharge. Duct papilloma meanwhile is frequently unilateral, single duct, yellow or clear (characteristically sometimes crystal clear) discharge with a watery consistency. Both can give blood staining, though this is more common with duct papillomas. The diagnostic problem, however, is that most clinicians would like something more than their clinical examination to make a definitive diagnosis. Mammography and ultrasound rarely show any changes in patients with periductal mastitis and do not have the resolution to identify the majority of duct papillomas. Although the majority of patients with nipple discharge and malignancy will have an associated palpable mass or a mass lesion/calcifications on mammography or ultrasound, a small number do not. Holland et al. demonstrated that only $50 \%$ of micropapillary or cribriform ductal carcinoma in situ (DCIS) is associated with microcalcification, and that in $70 \%$ of cases in situ malignancy in the region of the nipple is mammographically occult [3]. Clinical studies have found that between 3 and 9\% of patients with nipple discharge and no other clinical or imaging abnormality have an underlying malignancy [4-6]. The presence of blood staining increases the risk to $10-15 \%$, but the absence of blood does not exclude a malignant cause. Nipple discharge cytology occasionally shows frankly malignant cells in patients with DCIS or invasive malignancy, but its sensitivity is so low that a negative smear has no useful clinical meaning.

It is because of the inability of modern breast imaging to identify with certainty the cause of nipple discharge in most patients that surgeons generally advise the excision of the discharging duct (microdochectomy) for patients with unilateral symptoms. A microdochectomy typically removes the proximal $3-4 \mathrm{~cm}$ of the offending duct, excising and diagnosing duct papillomas with more certainty and often correctly identifying atypical epithelial proliferations that were otherwise clinically and radiologically occult. Surprisingly, a cause for the discharge is not found in approximately $20-25 \%$ of cases. This is partly because pathologists have no training or encouragement to carefully describe benign degrees of duct inflammation, with the consequence that the most common cause of nipple discharge often goes unmentioned or unrecognized after diagnostic surgical excision. The other reason is that microdochectomy is a completely blind surgical procedure. The discharging duct is cannulated (if it is still discharging) and dissected to a depth of 3-4 cm, without having any idea of the location of the intraduct pathology. Long-term follow-up shows that preinvasive malignancy and significant atypia are sometimes missed [7].

Breast duct microendoscopy (BDME) was pioneered in Japan, with the first descriptions appearing in Japanese, and the first in English in 1991 [8]. It was developed as a method for evaluation of nipple discharge and has been widely adopted in Japan and, more recently, in China. There is now considerable experience with the technique in the USA and Europe [9]. Large clinical series looking at women with nipple discharge have

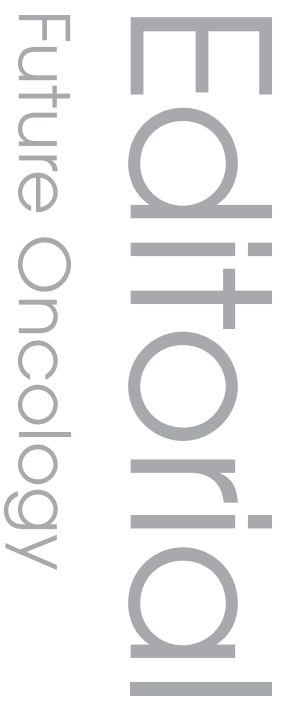

\section{fulure Medine $_{\text {mess }}$}


proven that duct microendoscopy can identify the cause of nipple discharge in as many as $80-90 \%$ of cases [10], and can diagnose atypical epithelial proliferations such as DCIS and atypical ductal hyperplasia. In a series of 415 patients with nipple discharge, Shen et al. found that the technique had a positive predictive value of $83 \%$ [11]. A total of $40 \%$ of patients had duct papillomas, but 11 had DCIS (2.7\%), of whom six had a normal clinical exam and breast imaging. Dooley identified ductal pathology in $96 \%$ of patients with bloodstained nipple discharge [12]. Two out of 27 patients had DCIS, and in both cases this was located more than $5 \mathrm{~cm}$ from the nipple so that it would have probably been missed by microdochectomy. In a third of his patients atypical ductal hyperplasia was demonstrated to co-exist with duct papillomas. Kaphenas-Valdes et al. found malignant lesions causing nipple discharge at an average of $4.4 \mathrm{~cm}$ from the nipple tip [6]. Direct comparisons of BDME and ductography have shown that ductoscopy is considerably more sensitive for the detection of intraductal pathology [13]. Liu et al. demonstrated that in patients with nipple discharge, BDME is more sensitive in detecting malignancy than mammography and ultrasonography combined [1]. Figures for the sensitivity of BDME alone in identifying malignant disease are encouraging. Matsunaga et al. studied 315 Japanese women with nipple discharge and found visible intraductal abnormalities in 80.9\% who had malignant disease [14], and a larger study by Liu et al. had a sensitivity of $94 \%$ [1]. It is inevitable that the sensitivity will be affected by the impossibility of examining every branch of an extensive duct system, which is probably the reason why easily recognizable lesions such as intraduct papillomas are not always seen. Shen et al. failed to identify $18 \%$ of benign papillomas that were subsequently shown on histological section [15]. However, the same study demonstrated the equal fallibility of surgical excision and microscopy, which failed to recognize any ductal pathology in $17 \%$ of cases with clear visible changes on microendoscopy.

\section{"Direct comparisons of BDME and ductography have shown that ductoscopy is considerably more sensitive for the detection of intraductal pathology."}

There is now convincing evidence that BDME provides an effective and noninvasive way to make a more definitive diagnosis in patients with nipple discharge. In a situation where current diagnostic tests are particularly unhelpful, and where there is a low but important risk of malignancy, BDME provides a specific diagnosis in a high proportion of patients. Even the recognition of duct inflammation as a manifestation of periductal mastitis is reliably identified by duct endoscopy [Pers. Commun.]. The less than perfect sensitivity for the diagnosis of malignancy means that in higher risk cases, such as blood staining or age over 50 years, BDME should be an adjunct to microdochectomy. In lower risk patients endoscopic evidence of benign intraductal pathology should be enough to safely avoid surgery. A recent series of 130 patients with intraductal papillary lesions found that there were no cases of malignancy in the 52 women less than 50 years of age [16], implying that it should be safe to omit microdochectomy in younger women if a duct papilloma(s) is definitely identified at endoscopy. This is important as duct surgery in young women can cause sufficient damage as to permanently prevent breastfeeding. Liu et al. avoided surgery in nearly 500 patients of all ages who had a negative endoscopy and aspirate cytology, and after 19 months of follow-up has missed only one case of DCIS [1]. However, even in higher risk cases BDME makes an invaluable addition to mandatory surgery by converting the procedure from a blinded one to an operation that is visually guided. Ling et al. have demonstrated that the addition of ductoscopy increased the detection of papillary lesions at microdochectomy from 40.7 to $90.6 \%$ [17].

A different use of BDME is in the intra-operative assessment of surgical margins in patients undergoing breast-conserving surgery. Dooley has successfully used this method to reduce the incidence of re-excision. The technique involves cannulating the mammary duct at the nipple and visualizing the duct epithelium between the nipple and the primary cancer. In more than 200 patients the primary lesion was identified in $75 \%$ of cases. In $41 \%$ atypia, in situ or invasive malignancy was detected more than a centimeter away from the primary lesion that had not been recognized preoperatively [18,19]. As a consequence, there were no patients that had a histologically positive margin on the nipple side of the excision, and overall, further surgery was needed in only $5 \%$ of cases as compared with $25 \%$ in patients in whom endoscopic evaluation had not been possible. However, these findings have not been easily reproduced. Kim et al. investigated 30 women undergoing breast conserving surgery 
and found nonspecific intraductal changes that were unhelpful in making accurate margin assessments [20].

Undoubtedly the most significant potential clinical application for BDME is for the early detection of radiologically occult duct epithelial atypia and in situ malignancy. It is currently believed that all patients with invasive mammary carcinoma have preceding epithelial atypia. Unless there is secondary microcalcification these changes are undetectable by any imaging technique. The progression to invasive malignancy takes many months and often years, providing a window of opportunity for the early detection and prevention of malignancy. BDME is unique in so much as it is the only way to directly access the duct epithelium. The technique is not envisaged to be a means of population screening and is really only appropriate for high-risk individuals, but does it have the potential to prevent invasive cancer by early diagnosis? Currently there is very little evidence to suggest whether this is a realistic possibility or a 'pipe dream'. Obtaining good intraductal histological specimens is still a problem that hampers the diagnosis of atypia. BDME provides good cytological samples in more than $80 \%$ of patients [10], and morphological assessment of these samples has been reported as being sensitive for malignancy in as many as $55 \%$ of individuals with cancer with a specificity of $100 \%$ [21]. More sophisticated analysis of cytological samples is one way of enhancing the diagnostic accuracy of BDME [22]. Fackler has used a quantitative PCR assay for the hypermethylation of the promoters of the RASSF1A, TWIST1 and HIN1 genes to look for malignant cells. In cytology samples obtained by BDME, this technique had a sensitivity of $100 \%$ and a specificity of $72 \%$ [23]. These early results suggest a role for ductoscopy in high-risk screening, but clinical studies on at-risk populations have yet to be completed.

"BDME remains the only method of
direct access to the duct epithelium and
will continue to present unique
opportunities for the study, diagnosis and
treatment of preinvasive malignancy."

The miniaturization required for BDME has been a technological challenge, which continues to delay the development of its use. Biopsy forceps capable of obtaining adequate histological specimens are still imperfect, although good results using a vacuum needle have been reported [24]. The problem of the sterilization of the very narrow internal channels $(0.25-0.5 \mathrm{~mm})$ of breast endoscopes has been overcome by modular endoscope construction. Nonetheless, the procedure is relatively simple, and can be carried out under local anesthesia. The endoscopes are not expensive unless completely disposable instrumentation is chosen.

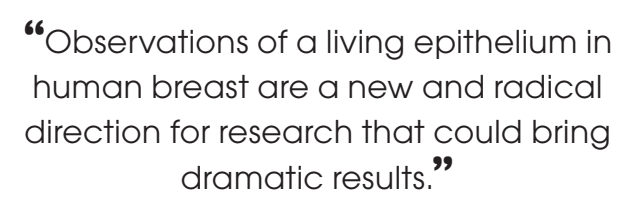

The technology that is involved in BDME has slowly improved so that definite indications for its use are now emerging. It is undoubtedly the most sensitive diagnostic tool for the investigation of patients with nipple discharge, and is invaluable as an adjunct to duct surgery. A much more significant role may be found in highrisk cancer screening, particularly when more advanced cell diagnostics are incorporated into the technique. BDME remains the only method of direct access to the duct epithelium and will continue to present unique opportunities for the study, diagnosis and treatment of preinvasive malignancy. As a research tool it is a technique that offers a completely new angle for the study of the breast epithelium in vivo. Repeated observation of the same area of duct epithelium is possible using metallic intraductal markers [ВевснеуNewman N. Pers. Observ.] and could facilitate the in vivo study of human preinvasive epithelial proliferations under different influences and over prolonged time periods. Observations of a living epithelium in human breast are a new and radical direction for research that could bring dramatic results.

\section{Financial \& competing interests disclosure}

The author has no relevant affiliations or financial involvement with any organization or entity with a financial interest in or financial conflict with the subject matter or materials discussed in the manuscript. This includes employment, consultancies, honoraria, stock ownership or options, expert testimony, grants or patents received or pending, or royalties.

No writing assistance was utilized in the production of this manuscript. 


\section{Bibliography}

1. Liu GY, Lu JS, Shen KW et al.: Fiberoptic ductoscopy combined with cytology testing in the patients of spontaneous nipple discharge. Breast Cancer Res. Treat. 108(2), 271-277 (2008).

2. Louie LD, Crowe JP, Dawson AE et al: : Identification of breast cancer in patients with pathologic nipple discharge: does ductoscopy predict malignancy? Am. J. Surg. 192(4), 530-533 (2006).

3. Holland R, Hendriks J, Verbeek A, Mravunac M, Schuurmanns Stekhoven J: Extent, distribution, and mammographic/ histological correlations of breast ductal carcinoma in situ. Lancet 335, 519-22 (1990).

4. Dietz JR, Crowe JP, Grundfest S, Arrigain S, Kim JA: Directed duct excision by using mammary ductoscopy in patients with pathologic nipple discharge. Surgery 132(4), 582-587 (2002).

5. Denewer A, El-Etribi K, Nada N, El-Metwally M: The role and limitations of mammary ductoscope in management of pathologic nipple discharge. Breast J. 14(5), 442-449 (2008).

6. Kapenhas-Valdes E, Feldman SM, Cohen JM, Boolbol SK: Mammary ductoscopy for evaluation of nipple discharge. Ann. Surg. Oncol. 15(10), 2720-2727 (2008).

7. Nelson RS, Hoehn JL: Twenty-year outcome following central duct resection for bloody nipple discharge. Ann. Surg. 243(4), 522-524 (2006).

8. Okazaki A, Okazaki M, Asaishi K et al:: Fibreoptic ductoscopy of the breast: a new diagnostic procedure for nipple discharge. Jpn. J. Clin. Oncol. 21, 188-196 (1991).
9. Kothari A, Beechey-Newman N, Kulkarni D: Breast duct micro-endoscopy: a study of technique and a morphological classification of endo-luminal lesions. Breast 15(3), 363-369 (2006).

10. Vaughan A, Crowe JP, Brainard J, Dawson A, Kim J, Dietz JR: Mammary ductoscopy and ductal washings for the evaluation of patients with pathologic nipple discharge. Breast J. 15(3), 254-260 (2009).

11. Shen KW, Wu J, Lu JS et al.: Fiberoptic ductoscopy for breast cancer patients with nipple discharge. Surg. Endosc. 15, 1340-1345 (2001).

12. Dooley WC: Routine operative breast endoscopy for bloody nipple discharge. Ann. Surg. Oncol. 9(9), 920-923 (2002).

13. Yamamoto D, Shoji T, Kawanishi $\mathrm{H}$ et al.: A utility of ductography and fiberoptic ductoscopy for patients with nipple discharge. Breast Cancer Res. Treat. 70 (2), 103-108 (2001).

14. Matsunaga T, Ohata D, Misaka T et al.: Mammary ductoscopy for the diagnosis and treatment of intraductal lesions of the breast. Breast Cancer 8, 213-221 (2001).

15. Shen KW, Wu J, Lu JS et al.: Fiber optic ductoscopy for patients with nipple discharge. Cancer 89 (7), 1512-1519 (2000).

16. Sakr R, Rouzier R, Salem C et al.: Risk of breast cancer associated with papilloma. Eur. J. Surg. Oncol. 34, 1304-1308 (2008).

17. Ling H, Liu GY, Lu JS et al.: Fiberoptic ductoscopy-guided intraductal biopsy improve the diagnosis of nipple discharge. Breast J. 15(2), 168-175 (2009).

18. Dooley WC: Endoscopic visualization of breast tumours. JAMA 284,1518 (2000).
19. Dooley WC: Routine operative breast endoscopy during lumpectomy. Ann. Surg. Oncol. 10(1), 38-42 (2003).

20. Kim JA, Crowe JP, Woletz J, Dinunzio A, Kelly T, Dietz JR: Prospective study of intraoperative mammary ductoscopy in patients undergoing partial mastectomy for breast cancer. Am. J. Surg. 188(4), 411-414 (2004).

21. Sauter ER, Klein-Szanto A, Macgibbon B, Ehya H: Nipple aspirate fluid and ductoscopy to detect breast cancer. Diagn. Cytopathol. (2009) (Epub ahead of print).

22. Fackler MJ, Rivers A, Teo WW et al.: Hypermethylated genes as biomarkers of cancer in women with pathologic nipple discharge. Clin. Cancer Res. 15(11), 3802-3811 (2009).

23. Cazzaniga M, Decensi A, Bonanni B, Luini A, Gentilini O: Biomarkers for risk assessment and prevention of breast cancer. Curr. Cancer Drug Targets 9(4), 482-499 (2009).

24. Hünerbein M, Dubowy A, Raubach M, Gebauer B, Topalidis T, Schlag P: Gradient index ductoscopy and intraductal biopsy of intraductal breast lesions. Am. J. Surg. 194(4), 511-514 (2007).

\section{Affiliation}

- Nicolas Beechey-Newman, BSc, MB, BS, FRCS, MS

The Harley Street Breast Clinic, 148 Harley Street, London, W1G 7LG, UK Tel.: +442079086071 Fax: +442079356423 nbn@sky.com 